\title{
Le temps des convertis, signe et trace de la modernité religieuse au début du $\mathrm{Xx}^{\mathrm{e}}$ siècle
}

Frédéric Gugelot

\section{OpenEdition \\ Journals}

Édition électronique

URL : http://journals.openedition.org/assr/1724

DOI : $10.4000 /$ assr. 1724

ISSN : $1777-5825$

Éditeur

Éditions de l'EHESS

Édition imprimée

Date de publication : 1 juillet 2002

Pagination : 45-64

ISBN : 2-222-96721-X

ISSN : 0335-5985

\section{Référence électronique}

Frédéric Gugelot, "Le temps des convertis, signe et trace de la modernité religieuse au début du Xx siècle ", Archives de sciences sociales des religions [En ligne], 119 | juillet - septembre 2002, mis en ligne le 18 novembre 2005, consulté le 01 mai 2019. URL : http://journals.openedition.org/assr/1724 ; DOI : $10.4000 /$ assr. 1724 


\section{LE TEMPS DES CONVERTIS, SIGNE ET TRACE DE LA MODERNITÉ RELIGIEUSE AU DÉBUT DU XX' SIÈCLE}

Entre 1885 et 1935, un mouvement de conversion au catholicisme se dessine au sein du milieu intellectuel (1). Nombre de contemporains en notent l'émergence, soit pour le célébrer, soit pour le regretter. Le romancier François Coppée, lui-même converti, est un des premiers à s'en réjouir : "Dans la tempête d'impiété qui sévit sur la France et quand les malfaiteurs qui la gouvernent s'efforcent de détruire dans l'âme du peuple jusqu'au dernier vestige du sentiment religieux, nous avons du moins une consolation, le retour pur et simple à la vérité chrétienne d'hommes d'élite, d'esprits très remarquables à divers titres, les uns par la force de la pensée, les autres par le don de l'imagination»(2). En 1912 en revanche, Marcel Sembat s'en inquiète : «Il y a (des) périodes où le vent souffle pour l'Église et il semble que nous soyons tout près d'une de celles-là. Le sentiment religieux est en grande ferveur dans les cercles poétiques et les jeunes revues littéraires. Surtout, et ceci est un symptôme très significatif et un indice révélateur, la libre-pensée, n'excite plus dans ces milieux aucun enthousiasme (...) Quand ce vent religieux souffle en littérature c'est qu'il souffle aussi dans les domaines de la pensée et de la vie sociale» (3). Son appréhension tient à ce que ce mouvement littéraire n'est pour lui que l'avant-garde d'une tendance de revanche du spirituel plus profonde et plus large au sein de la société alors que la séparation de l'État et des Églises semblait offrir l'étape ultime de la lutte contre l'obscurantisme. Ce changement d'identité spirituelle est en effet perçu comme un défi lancé à l'autre camp. C'est aussi pour la foi d'accueil, un véritable manifeste.

(1) Frédéric Gugelot, La conversion des intellectuels au catholicisme en France 1885-1935, Paris, CNRS Éditions, 1998 (cf. Arch. 108.49).

(2) François CoppéE, «Préface à Adolphe Retté», Du Diable à Dieu, Paris, L. Vanier, 1907, p. V.

(3) Marcel Sembat, «Un chef d'œuvre ami», Annales des fêtes et cérémonies civiles, juill.-août 1912, pp. 325-326. L'anticléricalisme de Sembat s'exprime dans son refus de mettre une majuscule à Église. 
Les années 1910 voient la question de la conversion devenir un objet d'actualité et de débat. De nombreuses traces en témoignent: la presse et le monde de l'édition prennent en compte l'événement. Les journaux titrent par exemple, faussement, sur la conversion de Barrès (4).

Quelques figures émergent et sont promues à l'avant-scène. Il s'agit de Paul Claudel, Charles de Foucauld, Charles Péguy et Jacques Maritain. Le phénomène ne se réduit néanmoins pas à ces quelques noms porte-étendard.

Or dans les années 1930, la figure du converti s'estompe au profit de celle du militant. Non que la conversion disparaisse, elle reste une réalité, une des formes d'adhésion et d'engagement religieux du XX $x^{\mathrm{e}}$ siècle. Mais le converti n'est plus sur le devant de la scène. Il y eut donc bien un «temps des conversions » où la figure du converti prit un sens et une valeur particuliers.

\section{Conversions et convertis}

La conversion appartient à l'histoire du christianisme, elle est inhérente à la nature prosélyte du catholicisme, mais rare à certaines époques où chacun, selon l'expression de Descartes, suit tout naturellement la religion de sa nourrice, elle révèle à d'autres un climat d'inquiétude religieuse (5). Il en est ainsi des dernières années du XIX et du début du XX $\mathrm{XX}^{\mathrm{e}}$ siècle.

Le converti Joseph Odelin n'hésite pas à réserver à la foi chrétienne le mot de conversion : "Qu'est-ce qu'une conversion? Cum vertere : tourner, changer, passer d'une fausse religion à la vraie, de l'incrédulité à la foi ; revenir de l'apostasie à la vie chrétienne» (6). Il n'existe pour lui qu'un seul sens au terme, celui qui va de l'erreur au catholicisme. Il est néanmoins vrai que la conversion veut étymologiquement dire orientation nouvelle, c'est-à-dire qu' «on se tourne vers un autre but ou un autre idéal que celui vers lequel on tendait jusque-là» (7). À la suite du $\mathrm{XVII}^{\mathrm{e}}$ siècle, toutes les définitions insistent sur le changement radical induit par la conversion, celui d'une mutation des idées et du mode de pensée (8). C'est donc le passage de l'incroyance à la foi ou le changement d'une foi à l'autre qui est étudié et non l'autre aspect possible, le retour constant de la foi.

(4) André Maurel, «La conversion de Maurice Barrès », Gil Blas, 30 mai 1912, 2 colonnes à la une et Paul Brulat, «Conversions religieuses », L'événement, samedi $1^{\text {er }}$ juin 1912, $1^{\text {ère }}$ page, 2 colonnes à la une.

(5) Nancy Gauthier, «La conversion au IV ${ }^{\mathrm{e}}$ siècle », in Michel Rouche, dir., Clovis, histoire et mémoire, Presses de l'Université de Paris-Sorbonne, 1997, p. 554. p. 32 .

(6) Joseph Odelin, Du théâtre à l'Évangile. Les étapes d'une conversion, Paris, Beauchesne, 1919,

(7) Dictionnaire de spiritualité, «Conversion», t. II, $2^{\mathrm{e}}$ partie, 1953, p. 2225.

(8) Pierre Dumonceaux, «Conversion, convertir, étude comparative d'après les lexicographes du $\mathrm{XVII}^{\mathrm{e}}$ siècle », La conversion au XVII ${ }^{e}$ siècle, 1983, p. 9. 
À l'époque contemporaine, en Europe occidentale, la conversion est une affaire personnelle (9). Il s'agit d'étudier «ce changement de croyance non l'objet du croire (un dogme, un programme, etc.)»(10), mais l'engagement d'un individu dans cette croyance, c'est-à-dire pénétrer l'intime de parcours spirituels aux résonances collectives.

Le prisme a changé et c'est un cheminement personnel qui est alors étudié et même dévoilé. Mais s'il concerne des individus, il n'en constitue pas moins un phénomène social, et donc un objet d'histoire : «La conversion est une forme d'accès à la foi qui éclaire singulièrement la nature de la foi» (11), note Maurice Nédoncelle. Quand de plus elle prend des allures de fait collectif, touchant une catégorie particulière de la société, les intellectuels, au moment même où ceux-ci obtiennent une visibilité sociale, dans le dernier quart du XIX $\mathrm{X}^{\mathrm{e}}$ siècle (12), ceci fait du phénomène de la conversion des intellectuels au catholicisme un objet de réflexion historique pertinent.

Pendant près de deux décennies, 1910 et 1920, ils vont hanter le catholicisme. Les trois principaux dictionnaires produits par le monde catholique dans la première moitié du siècle en témoignent a contrario. Le Dictionnaire de théologie catholique (13) n'inclut pas d'entrée conversion ou convertis lors de sa parution au début du siècle, de même que Catholicisme. Hier. Aujourd'hui. Demain en 1952 (14). En creux, s'inscrit une absence. Seul le Dictionnaire de spiritualité (15), débuté en 1932, comprend lui un article conversion long de 41 colonnes. Cet écart entre une actualité de la conversion et la faible trace qu'elle a laissée dans ces ouvrages, véritables bornes de la pensée catholique, montre bien que c'est une courte conjonction entre un temps historique, celui d'une citadelle assiégée, et un mouvement spirituel, qui semble répondre à une attente, qui a justifié la promotion du converti comme expression de l'actualité de la foi persécutée.

Quelle que soit la forme du parcours de ces convertis - les trois quarts d'entre eux étaient des catholiques de baptême - tous vivent les péripéties de leur périple spirituel comme une conversion pleine et entière. Même si seuls quelques athées, peu de protestants, moitié moins de musulmans et un nombre conséquent de juifs répondent exactement au schéma dominant. Tous révèlent que le catholicisme ne relève plus de l'acceptation passive mais d'un engagement personnel (16). À ce

(9) Dans le cadre des pays colonisés et en Europe orientale, lors de certains pogroms, on trouve des conversions de masse imposées par les événements. Il ne s'agit aucunement de ceci ici. p. 260 .

(10) Michel de Certeau, L'invention du quotidien, t. 1 Arts de faire, Paris, Gallimard, 1990,

(11) Maurice Nédoncelle, R. Girault, J'ai découvert le Dieu vivant, Paris, Revue des Jeunes, 1952 , p. 32.

(12) Christophe Charle, Histoire sociale de la France au XIXe siècle, Paris, Seuil, 1991, pp. 267-275 et Naissance des "intellectuels " 1880 - 1900, Paris, Éditions de Minuit, 1990. 1911.

(13) Dictionnaire de théologie catholique, A. Vacant, dir., continué par E. Mangenot, $2^{\mathrm{e}}$ tirage,

(14) Catholicisme. Hier. Aujourd'hui. Demain, G. JaCquemet, dir., Paris, Letouzey et Ané, 1952.

(15) Dictionnaire de spiritualité, fondé par M. Viller, F. CAVAllera, J. De Guibert S.J., continué par C. Baumgartner, S.J., Paris, Beauchesne, 1953, pp. 2224-2265.

(16) Jacques Julliard, "Naissance et mort de l'intellectuel catholique », Mil neuf cent, $\mathrm{n}^{\circ}$ 13, 1995, p. 10. 
titre, ils sont, sans le savoir, une figure de la modernité religieuse où l'emporte l'autonomie de l'individu-sujet (17).

Après les précurseurs des années 1885-1905, où se distinguent les noms de Paul Claudel, Charles de Foucauld, Joris-Karl Huysmans, François Coppée et Ferdinand Brunetière (par ordre chronologique), une véritable vague de conversions apparaît entre 1905 et 1915 . Cette décennie heureuse de la conversion diffère des précédentes par son ampleur et par sa variété. À quelques exceptions près, les précurseurs sont largement des "convertis de l'ordre». Ils cherchent dans la foi et l'Église un encadrement pour une société à leurs yeux en déliquescence. Plus âgés que leurs successeurs, ils reviennent à la foi de leur enfance mais surtout ils partagent une interprétation de l'histoire qui mêle condamnation des acquis de la Révolution de 1789 et aspiration à une société organisée par la morale chrétienne. L'Église leur apporte la pierre finale à un édifice de conservation sociale et de retour en arrière politique.

Si les convertis de 1905 à 1915 partagent ces opinions, ils n'apparaissent pas que comme des héritiers. Deux groupes d'âge différents sont perceptibles; un premier ressemble beaucoup à la majorité des précurseurs par son âge, ses origines idéologiques et ses vues politiques. Un deuxième groupe, les convertis en quête d'idéal, est plus jeune; ses membres ont entre 20 et 40 ans. Même s'ils partagent des idées politiques et religieuses proches du groupe des anciens, ils proviennent souvent de la gauche. Dreyfusards, socialistes, ces jeunes sont en quête d'un idéal de combat. L'affaiblissement de la mystique politique à la suite de la victoire du camp dreyfusard les mène souvent à chercher ailleurs un enthousiasme. Ils se rapprochent alors du nationalisme et de l'Action française. Mais les raisons politiques ne sont pas les seules. Un élitisme certain, mêlant rejet des générations précédentes et quête d'une morale de vie fondée spirituellement, joue. Jacques Maritain, Louis Massignon, Henri Massis, Ernest Psichari émergent de ce groupe. Mais bien d'autres noms, Henri Charlier, Emmanuel Delbousquet, Jacques Froissart, Henri Ghéon, Olivier-Hourcade, André Lafon, Paul Lœwengard, Joseph Lotte, montrent que l'Église dispose encore de messagers et de créateurs à opposer aux tenants de l'athéisme. Ils marquent leur époque en trouvant dans la guerre qui débute l'exutoire et la réalisation de leurs conceptions. Les vides qu'elle fait dans leurs rangs, la longueur du conflit, brisent le mouvement.

Le début des années vingt marque un étiage des conversions. Une reprise se dessine après 1925 et marque la fin des années vingt. Mais le nombre des conversions est moins important et les échecs moins rares, comme le montrent les conversions avortées de Jean Cocteau et de Maurice Sachs. D’ailleurs, les visages qui occupent le devant de la scène sont ceux des convertis d'avant la guerre, Maritain entre autres (18).

Ces trois temps de la conversion ne voient donc triompher la figure du converti que dans les années 1910. Elles ne sont pas isolées historiquement mais dépendent largement d'un premier terreau mis en place auparavant. De même, elles ne furent

(17) Danièle Hervieu-LÉGer, Le pèlerin et le converti, Paris, Flammarion, 1999, p. 30 (cf. Arch. 108.50).

(18) Au même moment, leur influence est aussi perceptible sur la vie intellectuelle américaine. Patrick AllitT, Catholic converts, Ithaca, Cornell University Press, 1997, p. 216. 
pas sans postérité. Mais ces quelques années cristallisent la figure du converti et son usage religieux ou social.

Des résistances sont alors perceptibles jusqu'au sein de l'Église. Elles permettent de saisir tout à la fois la promotion de cette nouvelle représentation du croyant et le rôle qui lui fut dévolu. Deux visions du catholicisme s'affrontent, deux images de sa place au sein de la société. La correspondance de l'abbé Frémont avec l'abbé Mugnier témoigne que dès l'origine du mouvement des phénomènes de rejet sont présents. L'abbé Frémont s'en prend d'abord au style littéraire de ces nouvelles ouailles : "“À l'homme qui scandalise un seul de ces petits, il vaudrait mieux qu'on lui suspendît au cou une meule de moulin et qu'on le jetât dans la mer." C'est Notre-Seigneur qui a dit cela, et ni Gury, ni Liguori, ni Pélion, ni Ossa, ni le collège romain échafaudé sur Saint-Sulpice ne détruiront cette ineffable et toute puissante parole» (19), écrit avec violence le premier au second. Et c'est ici au style réaliste de Huysmans que s'en prend le bon abbé. C'est que ces convertis concilient de façon extrêmement complexe conformisme religieux et des formes de modernité esthétique. Mais le reproche essentiel suit : «Le bon sens le plus élémentaire demande que des pécheurs qui, dans les premiers siècles du christianisme, auraient subi sept ans de pénitence aux portes de nos temples, ne soient pas maintenant, et du jour au lendemain, considérés comme des régulateurs et des justiciers, encore moins comme des gonfaloniers théologiques ou littéraires. Nul de nous ne doit faire témérairement de ces hommes je ne sais quels "vases d'élection", aussi longtemps que ces hommes eux-mêmes n'auront pas brisé courageusement leurs "vases d'ignominie", et qu'ils continueront à vivre de leurs romans lamentables et à mépriser les formelles et divines injonctions de Notre-Seigneur : Si pes tuus scandalisaverit te, abscide eum et projice abs te» (20). Le reproche essentiel est la place que ces convertis tendent à acquérir au sein de l'Église alors même qu'ils ne sont que des catholiques de fraîche date. Ils sont d'ailleurs objet d'affrontement entre ordres religieux, les dominicains orchestrant une partie de l'affaire, les jésuites tentant de la noyer dans la masse. Ainsi au sein du Dictionnaire de spiritualité, le phénomène est resitué dans une histoire et une pensée longue par le jésuite Henri Pinard de la Boullaye (21) dans une nette volonté de le restreindre autant que faire se peut. Il n'hésite pas, dès les premières lignes, à montrer à quel point il porte peu de considération à la littérature de convertis : "Ces quelques distinctions laissent entrevoir à quelle critique prudente il convient de soumettre, aussi bien que les confidences orales des "convertis" (on notera les guillemets), leurs apologies personnelles et leurs autobiographies, les récits hagiographiques exposant les succès des convertisseurs et les descriptions littéraires d'initiations » (22). Au-delà de ces critiques, le simple fait de resituer dans une histoire longue le phénomène, ainsi que le fait un autre jésuite, Joseph Huby, permet de le nuancer et donc d'en limiter la portée. Ses mots sont aussi durs : «Préoccupés d'atteindre ceux du dehors, plu-

(19) Lettre de l'abbé Frémont à l'abbé Mugnier du 10 mars 1898, Bulletin de la Société Joris-Karl Huysmans, $\mathrm{n}^{\circ}$ 9, 1933, p. 63.

(20) Lettre de l'abbé Frémont à l'abbé Mugnier du 18 mars 1898, Ibid., p. 64.

(21) Au carême 1928, Mgr Baudrillart remplace le P. Sanson comme prédicateur à Notre-Dame, ce dernier étant la voix du P. Laberthonnière. C'est Pinard de la Boullaye qui prend la suite du recteur de l'Institut catholique.

(22) Henri Pinard de la Boullaye, «Conversion», Dictionnaire de spiritualité, op. cit., p. 2225. 
sieurs (convertis) n'ont pas assez songé à ceux du dedans et se sont installés dans la maison du Père de famille un peu trop comme en pays conquis $\gg(23)$. Les reproches énoncés par Frémont se retrouvent parfaitement ici sous la plume d'Huby. Les convertis, hantés par leur perte de foi, pleins de la joie de leur découverte, tentent de reconstruire une apologétique de combat dont l'objectif est bien d'atteindre les non-croyants extérieurs. Ces critiques condamnent la mission qu'ils se sont attribuée et les moyens qu'ils utilisent. L'éthique chrétienne a pourtant toujours privilégié la conversion d'un pécheur à la fidélité du juste (24). Huby, lui, gère et défend les citadelles du classicisme intellectuel : «Comment par exemple s'étonner que d'excellents catholiques puissent être blessés d'entendre un converti d'hier ou d'avant-hier clamer qu'un officier, un prêtre peuvent être des épiciers selon l'esprit et le sont malheureusement presque tous, comme si l'esprit chrétien ou même l'esprit tout court se mesurait nécessairement au mépris pour l'Académie française et à l'admiration pour tel ou tel littérateur qui n'en fit pas partie» (25). Au-delà de l'affrontement littéraire qui en filigrane parcourt ces remarques acerbes, c'est déjà en germe la question de savoir si la France est une terre de mission ou non. Les convertis savent, eux, que la foi est menacée, leur vie même en est la preuve. D'où l'actualité de leur cheminement et la nécessaire publicité de celui-ci.

Or c'est au moment où l'intellectuel «naît» socialement que certains catholiques prennent conscience de leur caractère minoritaire au sein de la société française (26). Ce constat, le converti Jean de Beaucorps le dresse : «Est-ce que chacun ne sait pas (...) et ne s'en va pas répétant que la religion se meurt, que la grâce se retire du monde, que la foi baisse à mesure que la science se hausse, que le doute nous envahit, que la puissance de Dieu semble subir une éclipse, que notre siècle de matérialisme ne produit plus ni saints ni miracles?» (27). Cette prise de conscience fonde la nécessité impérieuse de la publicité faite à nombre de conversions. C'est au nom de cette sécularisation que le catholique intellectuel se doit d'intervenir dans le champ politique et social. La multiplication des récits de conversion s'explique donc par l'actualité, puisqu' «En nos jours, plus que jamais, la Vérité catholique a besoin d'interprètes et de défenseurs compétents» (28). Ce mouvement prit des couleurs collectives et pût être perçu et présenté comme tel. Cette dichotomie entre passage individuel et phénomène collectif s'inscrit dans les variantes de l'engagement des convertis. Certains restent discrets sur leur nouvelle foi; d'autres, au contraire, deviennent des figures de proue. C'est bien cette mise en avant, non de la conversion elle-même, mais du converti, qui est la nouveauté de ce début de siècle. Or «ce qui caractériserait l'intellectuel, c'est le va et vient, la relation consubstantielle qu'il opère entre ses choix esthétiques et ses choix idéologiques que ce soit sur le mode de l'académisme ou de l'avant-garde : l'activité intellectuelle consiste en quelque sorte à ne cesser d'éclairer et de justifier l'un par

(23) Jospeh Huby, «Le témoignage des convertis », Études, t.155, 1918, p. 395.

(24) Bernard Pouderon, «La conversion chez les apologistes grecs», in Jean-Claude Attias, dir., De la conversion, Paris, Cerf, 1997, p. 154.

(25) Jospeh Huby, «Le témoignage des convertis», op. cit., p. 395.

(26) Jacques Julliard, op. cit, p. 6.

(27) Jean de Beaucorps, Lourdes, t. 2, Les guérisons, 1913, p. 373.

(28) Théodore Mainage, O.P., rédacteur en chef de la Revue des Jeunes, Introduction, Les Témoins du Renouveau Catholique, Paris, Beauchesne, 1917, p. 17. 
l'autre, et c'est en cela qu'il existe une relation structurelle entre les valeurs et les formes de sociabilités " (29). L'aspiration des convertis correspond à cette recherche. Ils quêtent un sens, une adéquation entre leurs idées, leurs œuvres et leur vie. Il est donc possible d'inclure l'élan personnel dans un phénomène historique à teneur plus large. Et parce que la mutation des pensées n'est alors parfois conçue qu'accompagnée d'un engagement public, le phénomène des convertis conjugue un double besoin : celui d'artistes ou d'écrivains nouveaux chrétiens qui font de la publicité de leur foi, et de leur art, expression de cette foi, un devoir, et celui d'une Église qui trouve en eux d'ardents défenseurs. Max Jacob a le mieux exprimé cette conjonction : "Le devoir moderne, le devoir actuel, presque notre seul devoir est celui de l'apostolat par l'exemple; quand nous avons un peu d'influence, affichons nos sentiments religieux, manifestons. Le temps n'est plus des fleurs d'ombres dans les Églises noires mais c'est le siècle de la publicité. Ferons-nous moins pour l'Art et pour la Foi que Cadum pour son savon?»(30). Le parallèle est osé mais expressif. Ils ne font que répondre à une pression de leur temps. «C'est donc la séparation de l'Église et de l'État (1905) (...) qui (...) détermine, autant que l'affaire Dreyfus, la naissance d'une intelligentsia catholique en France : désormais la foi est affaire individuelle; elle est par conséquent aussi affaire de "profession" publique $\gg(31)$.

Cela se traduit dans l'orientation des formes apologétiques mises en place et renouvelées par eux.

\section{Manifeste et témoins}

La promotion de la figure du converti s'accompagne inévitablement de la promotion du récit de conversion au sein d'une littérature apologétique contestée (32). Ces écrivains - ce sont essentiellement eux qui témoignent - offrent leur cheminement en exemple de la faillite de l'apologétique du XIX ${ }^{\mathrm{e}}$ siècle. Il leur faut aussi conquérir ce nouveau champ et pour cela rejeter les anciennes productions. Ces nouveaux catholiques avancent deux raisons pour justifier l'écriture de tels récits. La première est de «rendre témoignage et crier sur les toits que tout est vide et vain auprès de la Gloire de Dieu et en dehors de la Croix de Notre-Seigneur Jésus-Christ» (33). La seconde est le désir d'édification et de diffusion de la foi nouvelle. C'est à l'aune du cheminement spirituel que les événements sont jugés, catalogués et décrits.

(29) Michel Trebitsch, «Avant-propos : la chapelle, le clan et le microcosme», Sociabilités intellectuelles. Lieux, milieux, réseaux, Cahiers de l'I.H.T.P., no 20, mars 1992, p. 20. p. 59.

(30) Max JАсов, Lettres à Marcel Jouhandeau, Lettre du 12 juillet 1927, Genève, Droz, 1979,

(31) Jacques Julliard, op. cit., p. 10.

(32) Voir sur l'écriture de l'histoire sainte du Xxe siècle, l'article de Fabrice Bouthillon, «Comment on écrit l'histoire sainte. À propos d'une vie de Marthe Robin », Revue des sciences philosophiques et théologiques, t. 74, $\mathrm{n}^{\mathrm{o}} 4$, oct. 1990, pp. 537-565.

(33) Pierre Van der Meer, Journal d'un converti, Paris, Téqui, 1921, p. 5. 
La simultanéité de la crise entre l'État et l'Église et de l'émergence du groupe des intellectuels multiplie les récits de conversion émanant de ceux qui deviennent des acteurs du jeu politique et social. L'Église sait que les intellectuels peuvent être de magnifiques vecteurs de son message au cœur de la société, au moment où leur rôle se développe.

Élément de l'écriture de l'histoire sainte du $\mathrm{XX}^{\mathrm{e}}$ siècle, le récit de conversion prend alors une valeur apologétique que l'Église, et le converti avec elle, ne peuvent négliger. Il prouve aussi l'acuité de l'inquiétude religieuse qui hante ce début de siècle. Monseigneur Baunard qui œuvre à la diffusion de ce qu'il intitule des victoires de la Foi, n'oublie pas de souligner l'intérêt de tels exemples : «Nous (...) continuerons par d'autres études semblables sur les plus grands convertis de l'époque présente, en ayant soin de les choisir parmi les rangs supérieurs de la hiérarchie sociale ou intellectuelle, afin que leur exemple ait d'autant plus d'autorité qu'il descend de plus haut» (34). Cette interprétation des conversions favorise évidemment l'écriture et la diffusion des récits de conversion. Ils sont la marque même d'un temps déchristianisé, au cours duquel la découverte ou la reconquête de la foi apparaissent comme des éléments apologétiques (35). L'ampleur des débats français autour de la question religieuse à la fin du XIX et au début du XX $\mathrm{XX}^{\mathrm{e}}$ siècle sont des facteurs déterminants, non seulement de la conversion, mais aussi de l'écriture du récit de ces conversions, comme le prouve la chronologie de la parution de la majorité de ces récits. Il s'agit d'une écriture de combat, de "guerre civile» (36) même pour François Coppée. Les années 1910 du siècle, climax du mouvement, sont aussi le moment où s'éditent le plus de récits. Adolphe Retté note dans un des premiers recueils de conversion portant sur la période contemporaine : «Il semble que pour l'avenir très sombre qui se prépare, Dieu se recrute de nouveaux combattants» (37). Il explique le but recherché : «Dans les pages qui suivent, j'ai coordonné et analysé plusieurs de ces récits de conversions. Si j’ai pu intéresser, peut-être Dieu daignera-t-il en user pour toucher quelques âmes encore errantes loin de lui » (38). L'écriture devient missionnaire et le converti, par sa production, participe de l'effort prosélyte. Dans le même temps, cette position est une critique de l'Église et de son clergé. Si le discours de propagande nécessite d'être renouvelé, c'est bien qu'il est défaillant. Et ces «convertis de l'intérieur», c'est-à-dire catholiques de baptême ayant perdu la foi avant de renouer avec elle, en ont conscience; ils ont souffert de cette absence de Dieu que leur périple pointe au cœur de la société. Leur position même condamne une Église du XIX ${ }^{\mathrm{e}}$ siècle qui ne trouve plus grâce à leurs yeux.

L'urgence de la relation se déduit de cette interprétation de l'époque comme celle d'une lutte à mort entre un catholicisme persécuté et un monde déchristia-

(34) Mgr Baxunard, La Foi et ses victoires, t. 1, Paris, De Gigord, 1919 (Préface datée de 1881, reprise en 1901), p. VI.

(35) Louis Pérouas note combien ce choix apologétique de l'exemplarité par en haut est partagée par de nombreux membres du clergé et a pu parfois être trompeur. Louis PÉROUAs, Refus d'une religion. Religion d'un refus en Limousin rural, 1880-1940, Paris, École des Hautes Études en Sciences Sociales, 1985, pp. 124-125.

(36) Paul Bourget, L'Étape, Paris, Plon, 1902, p. 506.

(37) Adolphe Retté, Quand l'Esprit souffle, Paris, A. Messein, 1914, p. 10.

(38) Ibid, p. 10. 
nisé (39), cela justifie l'écriture : «Je le fais avec d'autant plus de joie qu'il y a en ce moment quelque courage à oser affirmer ses croyances » (40). La multiplication des récits de conversion s'explique donc par l'actualité. Les références aux premiers chrétiens n'apparaissent pas comme un simple effet de style, il s'agit bien d'une lecture de leur temps. Ainsi les récits sont des armes et les auteurs les forgerons de la renaissance catholique : «Serions-nous à la veille d'une renaissance religieuse? Je suis de ceux qui le croient. Déjà, semble-t-il, la Providence se prépare partout pour cette restauration d'ardents ouvriers» (41).

Le R. P. Barge, directeur de la Revue des Jeunes en ces années 1913-1914, a bien conscience de l'importance de tels récits. Souhaitant utiliser au plan apologétique la renaissance catholique qui semble se dessiner dans les années 1910, il lance une enquête sur le renouveau catholique (42) où il souhaite insister sur un des signes de ce renouveau, le mouvement de conversion: "La Revue des Jeunes, postée en éclaireur aux avant-gardes de la génération nouvelle, a mis au nombre de ses tâches celle de recueillir les récits de conversion» (43). Le mot est révélateur, recueillir, il s'agit bien ici d'amasser en vue de conserver et d'utiliser ultérieurement. L'idée d'avant-garde est aussi significative. Fernand Lelotte résume ces arguments quand il présente l'apport de tel récit: «Jadis les chrétiens fortifiaient leur foi à la lecture des Passions des Saints. Pourquoi les récits de conversion ne joueraient-ils pas aujourd'hui un rôle semblable?» (44). Lelotte joue sur le sens du mot martyr qui en grec et en latin veut dire témoin. Puisque, jusque-là, l'Église a fait appel à leur exemple, dans la situation qui est maintenant la sienne, les martyrs sont devenus rares en Occident, les nouveaux témoins ne doivent-ils pas être recherchés dans ces nouveaux chrétiens? Car tout parcours peut offrir des lumières à d'autres. Deux visées se dégagent. La première, Pierre de Lescure l'énonce clairement : «Je parle, vois-tu, parce qu'il s'agit d'un plus Grand que moi qui est aussi ton Hôte, que tu le saches ou non » (45). Il faut prendre date, témoigner de l'existence de Dieu et de son action. Le deuxième but est de s'adresser aux incroyants et aux chercheurs de Dieu : «Et peut-être pourrai-je indiquer à quelques hommes qui errent et cherchent et meurent de soif, la source d'eau vive qui jaillit, là, devant les pieds meurtris et las de cheminer» (46). La recherche de l'exemplum est à la fois partagée par les auteurs et par les compilateurs. Ce double objectif pèse sur l'écriture du récit. "Soyez béni que le secret soit désormais le bien commun,» écrit Mireille Dupouey à Henri Ghéon à l'occasion de la parution de son récit de conver-

(39) C'est du moins l'idée qu'ils s'en font. Bien des travaux historiques récents prouvent que ce jugement est à nuancer fortement. Jacques Le GoFf, René RÉMOND, Histoire de la France religieuse, t. 3, XVIII $^{\mathrm{e}}$-XIX ${ }^{\mathrm{e}}$ siècle, Paris, Seuil, 1991, et Jacques GADILle, Jean-Marie MAYEur, dirs., Histoire du christianisme, Paris, Desclée, 1995, t. 11.

(40) A.A., «Mon retour à Dieu!», Revue de la Jeunesse, 25 mars 1914, p. 636.

(41) Louis Le Cardonnel, Mercure de France, $1^{\mathrm{er}}$ août 1907 reproduit dans De l'anarchie intérieure à l'union mystique, quelques témoignages sur A. Retté, Paris, A. Messein, 1957, p. 30.

(42) Rejoignant en cela la mode de l'enquête dont Agathon est l'exemple le plus célèbre. JeanJacques BECKER, «Présentation », in MASsis, DE TARDE, Les jeunes gens d'aujourd'hui, Paris, Imprimerie Nationale, 1995, p. 11.

(43) Théodore Mainage, op. cit., p. 20.

(44) Fernand Lelotte, Convertis du XXe siècle, t. 4, Paris-Tournai, Castermann, 1958, p. 6.

(45) Pierre DE Lescure, «Des «Religions de 1'Esprit» à la «Religion de l'Autorité»», in Théodore Mainage, Les témoins du Renouveau catholique, Paris, Beauchesne, 1917, p. 207.

(46) Pierre Van der Meer, Journal d'un converti, Paris, Téqui, 1921, p. 5. 
sion, L'homme né de la guerre (47). Jouant sur le sous-titre du livre, Témoignage d'un converti, Mireille Dupouey exprime parfaitement l'enjeu d'un tel texte. Léopold Levaux reprend un argument identique : "Jamais je n'ai pensé que ce procès-verbal, parfaitement spontané et sincère du drame intime de ma vingtième année serait lu et encore moins publié. S'il l'est aujourd'hui, c'est pour rendre témoignage» (48). Témoigner pour la foi et l'Église, livrer l'intime au public pour la vérité ? Claudel le montre bien dans sa lettre à Massignon du 17 novembre 1913 : «Le R. P. Barge, dominicain, directeur de la Revue de la jeunesse, publie en ce moment une série de récits de conversions. Il m'a demandé celui de la mienne, que je n'ai pas cru pouvoir lui refuser, malgré ma vive répugnance. Il paraît que ces récits emportent avec eux une grande force de conviction et d'entraînement (...) auriez-vous strictement le droit de répondre par un refus à une demande qu'on vous ferait? Croyez-vous que ce soit uniquement pour vous que vous ayez été converti?» (49). Claudel, bien que réticent à délivrer ce qu'il considère comme son expérience la plus secrète, s'est rangé aux arguments du dominicain. Le converti se doit de prouver l'action de Dieu par son existence et par son récit qui témoigne de l'action de la grâce, de la présence du surnaturel au cœur du XX $\mathrm{X}^{\mathrm{e}}$ siècle. À ses yeux, être converti, c'est surtout témoigner dans un monde qu'il croit déchristianisé : «Ceux qui, ayant reçu la lumière, ne s'efforcent pas comme désespérément de la propager autour d'eux (...) sont bien coupables» (50). Ailleurs il ajoute : "C'est toujours un miracle étonnant qu'une conversion, c'est une interpellation directe par laquelle Dieu s'adresse personnellement à nous et il est un artiste trop économe de ses moyens pour qu'aucun de ses rares coups de théâtre soit inutile à ses vastes plans» (51). Une fois de plus, est ici perceptible l'idée selon laquelle la conversion d'un intellectuel participe d'un plan divin, qui impose un devoir particulier. Claudel reprend l'argument auprès de Jammes : "Qu'il le veuille ou non, un converti est donc une preuve vivante, il est un témoin mis là pour qu'on ait recours à lui, il a le devoir de parler, et de répondre à ceux qui l'interrogent» (52).

C'est donc au nom de ce devoir de témoigner que les convertis justifient l'écriture du récit. «Chaque exemple, écrit Léon Moulin, apporte ainsi sa preuve, de si haut ou de si bas qu'il nous vienne, par suite peut-être utile à raconter» (53). Le but apologétique est bien le premier objectif de ces récits. Ce don de la foi, offerte par Dieu, le converti se doit de le délivrer par son récit: "J'ai été souvent invité de façon amicalement pressante, à écrire le récit de ma conversion. Écœuré devant l'abîme de détresse morale, de perverse corruption, d'agressive impiété où je me suis douloureusement débattu pendant trente ans, j'ai toujours repoussé comme intempestive et gênante cette sollicitation (...) Puissent le bon Dieu que j'aspire à servir en soldat indéfectible, et ma si généreuse Protectrice, sa sainte Mère,

(47) Mireille Dupouey, Lettres, Paris, Cerf, 1940, p. 26.

(48) Léopold Levaux, Quand Dieu parle, Paris, Bloud et Gay, 1926, p. XIII.

(49) Lettre de Claudel à Massignon du 17 nov. 1913 in Paul Claudel - Louis Massignon, Correspondance 1908-1914, Paris, Desclée, 1973, pp. 213-214.

(50) Paul Claudel - André Gide, Correspondance 1899-1926, Paris, Gallimard, 1949, p. 114.

(51) Lettre de Claudel à Massignon du 12 oct. 1908 dans Paul Claudel - Louis Massignon, op. cit., p. 50.

(52) Lettre de Paul Claudel à Francis Jammes du 26 mai 1911, Paul ClaUdel - Francis JAMMES, Gabriel Frizeau, Correspondance 1897-1938, Paris, Gallimard, 1952, p. 209.

(53) Charles de Bordeu, «Ma conversion», in Théodore Mainage, op. cit., p. 91. 
permettre à ces modestes lignes extraites du mémorial d'une âme contrite et débordante de gratitude, de faire quelque bien surtout aux égarés qu'aveugle le Génie du mal!»(54). Tous les enjeux de la campagne de la Revue de la Jeunesse sont ici résumés. Une fois vaincue la pudeur à voir livrée son expérience intime la plus ultime, le converti a bien conscience d'œuvrer à une apologétique de temps de crise. Aussi la sollicitation ecclésiastique est-elle ici décisive. Le combat est double, celui d'une Église assiégée, qui vit comme une persécution la loi de séparation, et celui de la mémoire. Le choix par l'auteur du terme "mémorial" montre bien cette volonté d'inscrire l'action de la grâce dans le siècle et dans le temps long de l'Église. La référence pascalienne ne surprend pas. Le récit de conversion est un monument élevé à la mémoire de l'action de la grâce. Allo est un de ceux qui inscrit le plus profondément son expérience de converti dans les enjeux de son temps. «J'ai écrit ces lignes pour que quelque attardé aux idées de ma génération, si elles lui tombent sous les yeux, voie comment Dieu a su prendre un prétentieux et antipathique nietzschéen, par son propre idéal de Force, pour en faire un catholique» (55). Il souhaite faire de son exemple un exemple pour l'ensemble de sa génération. N'a-t-elle pas vécu comme lui ce dégoût de soi, issu du nietzschéisme et du matérialisme? Le cadre historique explique l'actualité de la conversion et par là-même la large diffusion du récit de conversion. Néanmoins une transformation est perceptible en son sein.

Aux XIX et $\mathrm{XX}^{\mathrm{e}}$ siècles, la "rationalisation" qui tout à la fois est une doctrine, une vision du monde et une procédure mentale (56) conduit à un désenchantement $\mathrm{du}$ monde, une «individualisation» du croire et à une «privatisation du sentir» (57). Or de façon concomitante, ces temps sont aussi ceux de la prolifération des récits autobiographiques, formes laïcisées de la pratique de l'examen de conscience, véritables «baromètres de l'âme» (58). Alors que nombreux sont ceux qui signent leur récit par des initiales ou tout simplement, "Une convertie"; c'est le contraire qui se produit pour les convertis de la fin du XIX ${ }^{\mathrm{e}}$ et du début du $\mathrm{XX}^{\mathrm{e}}$ siècle. Car le nom, la valeur même des auteurs, leur rôle possible dans un contexte donné, justifient cette disparition des initiales ou de l'anonymat au profit $\mathrm{du}$ nom, devenu étendard. La figure de l'intellectuel modifie donc le récit de conversion valorisant l'identité de l'énonciateur au détriment de la teneur du message. Il permet à l'Église de contrer l'idéologie positiviste de la République en opposant aux grands hommes de culture et de science qu'elle met en avant, d'autres grands noms. Charles Morice justifie ainsi la rédaction de son récit. «Il ne m'est pas permis de servir la cause de Dieu en secret. Je dois m'employer pour sa gloire, selon le mode d'activité qui m'est prescrit par ma nature. Puisque ma fonction est de parler, je n'ai pas le droit de taire les raisons auxquelles j'ai cédé» (59). C'est bien son statut de professionnel de la prise de parole qui contraint Morice à témoi-

(54) A.A., op. cit., p. 636.

(55) Bernard-Marie Allo (sous la signature B. E.), «De Gœthe au Christ», in Théodore MaINAGE, op. cit., p. 204.

(56) Jean-Claude PASSERON, «L'espace wébérien du raisonnement comparatif», in Max WeBER, Sociologie des religions, Paris, Gallimard, 1996, p. 8.

(57) Marcel Gauchet, La religion dans la démocratie, Paris, Gallimard, 1998, p. 17. (cf. Arch. 116, pp. 41-52)

(58) Pierre Pachet, Les baromètres de l'âme. Naissance du journal intime, Paris, Hatier, 1990.

(59) Charles Morice, Lettres à mes amis sur quelques points de durable actualité. Le Retour ou Mes Raisons, Paris, A. Messein, 1913, p. 26. 
gner, c'est au titre d'intellectuel qu'il rédige le récit de sa conversion. «Puisqu'il n'existe de profitable leçon que la leçon vécue» (60), là réside selon André Godard l'intérêt de tel récit.

Le récit de conversion est une autobiographie spirituelle, une écriture de soi. La relation de chaque conversion est un témoignage personnel qui répond à cette question que Du Bos présente comme initiale et capitale dans son étude du récit de conversion de Claudel : "C'est parce qu'ils se meuvent au sein du brouillard que l'incrédule, l'indifférent et l'aveugle marchent sans savoir où ils sont et qui ils sont, et c'est parce qu'ils ne savent pas où ils sont qu'ils ne savent pas qui ils sont» (61). Le récit révèle les réponses découvertes par l'auteur et c'est à ce titre qu'il est jeté à la face des incroyants. Pour Joseph Huby, le récit de conversion du $\mathrm{XIX}^{\mathrm{e}}$ et du $\mathrm{XX}^{\mathrm{e}}$ siècle a, de ce point de vue, changé par rapport à ceux des siècles précédents. «Avec le dix-neuvième siècle, le genre va prendre une nouvelle extension, mais en se modifiant d'une façon sensible. Sans que le côté intellectuel soit supprimé, une large place est accordée à l'analyse psychologique, à la peinture des états d'âme. Les convertis ne se contentent plus d'exposer leurs raisons de croire. Ils se plaisent à retracer, étape par étape, l'itinéraire qu'ils ont suivi jusqu'au seuil de l'Église catholique»(62). Le récit de conversion est donc un enfant de son siècle. Il est la preuve de l'action de la grâce. «La sacralisation des pratiques littéraires, caractéristique de notre époque, semble donc avoir pour corrélât une expérience littéraire du sacré », écrit Jean-Pierre Albert (63). En un temps où l’Église se vit comme une citadelle assiégée, le récit devient la preuve de sa pérennité et de son actualité. Littérature de temps de crise, le récit de conversion est un élément de la tentative de reconquête des élites françaises par le catholicisme. Il est aussi un exemple d'adaptation de l'Église à l'affirmation du rôle des intellectuels en France. Ce nouvel ensemble, partiellement mobilisé contre elle mais également mobilisable pour elle, lui offre des voix nouvelles à faire entendre aux fidèles comme aux gens de l'extérieur. Le récit de conversion est l'une de ces voix. Tout comme la mystique du XVI $\mathrm{XIVII}^{\mathrm{e}}$ siècle prolifère autour d'une perte (64), celle d'un univers qui se défait, rendant lisible une absence, celle d'un monde uni sous une seule foi, cette écriture spirituelle du début du $\mathrm{XX}^{\mathrm{e}}$ siècle renvoie, elle, à un univers défait, rendant visible un monde sans foi. L'interrogation spirituelle habite alors de nombreuses œuvres et envahit la littérature profane. Le récit de conversion ne se cantonne plus à l'exemple des vies de saints, à l'édification des fidèles et à l'apologétique des mécréants; il prend pied dans l'expression du soi littéraire dans une évolution parallèle à celle de l'autobiographie et du journal personnel, qui d'œuvre secrète au $\mathrm{XIX}^{\mathrm{e}}$ deviennent une expression pleine et entière de l'écriture au $\mathrm{XX}^{\mathrm{e}}$.

Épanchement de soi, épanchement de l'âme sous des formes littéraires très diverses mais où le journal intime, moyen décisif d'introspection et d'expression du cheminement vers la foi, joue un rôle décisif. Il «est l'instrument approprié que se

(60) André Godard, In memoriam, Paris, Calmann Lévy, 1899, p. 53.

(61) Charles Du Bos, "Commentaires au bas d'un grand texte Ma conversion par Paul Claudel », (1934), Approximations, Paris, Fayard, 1965, p. 1175.

(62) Joseph Huby, «Le témoignage des convertis», Études, t. 155, 1918, p. 389. p. 81 .

(63) Jean-Pierre Albert, «Hagio-graphie. L’écriture qui sanctifie», Terrains, no 24, mars 1995 ,

(64) Michel de Certeau, La fable mystique, Paris, Gallimard, 1982, p. 25. 
suscite une certaine recherche théorique décidant de rompre avec la pensée systématique ou faisant porter ses efforts sur des états de subjectivité » (65).

L'œuvre autobiographique devient là un élément décisif du processus de conversion, un moyen crucial d'introspection et d'expression mais aussi de diffusion de son voyage vers la foi. Et résonne alors cette phrase de Mauriac qui pourrait s'appliquer à tous quel que soit le genre d'expression utilisé : «Tout auteur de mémoire, chacun à sa façon, et fût-ce en s'accusant, prépare sa défense... Devant la postérité? Peut-être, mais inconsciemment ne cherche-t-il pas à fixer l'aspect qu'aura son âme aux yeux de Celui qui la lui donna et qui peut la lui redemander à chaque instant?» (66). Toute écriture sur soi s'inscrirait donc dans un rapport avec Dieu (67) et cette tentation habite encore nombre d'œuvres du XX siècle (68). «Texte référentiel» (69) de forme, d'inspiration ou d'écriture autobiographique qui exprime cette recherche de soi et de l'Autre, le récit de conversion, à l'égal de la conversion, est alors un réenchantement du monde.

Cette production s'accompagne d'une ardente campagne de diffusion et de promotion, orchestrée par certains milieux ecclésiastiques. Il faut noter qu'au moment où le mouvement de conversion s'essouffle - à partir de la Première Guerre mondiale -, des ouvrages, de membres du clergé en particulier, relaient et diffusent ses résultats. Dès 1912, A. Dossat et J. Montjovet, rédacteurs à La Croix, rassemblent leurs articles consacrés aux plus célèbres convertis du temps (70). Adolphe Retté, lui-même converti, chante le «souffle de l'esprit»(71). Le Père Mainage reprend une partie des récits parus dans la Revue de la Jeunesse dans un ouvrage au titre révélateur, Les Témoins du Renouveau catholique (72). Monseigneur Chollet les utilise dans ses prêches édités peu après (73). L'abbé Joseph Lescure œuvre de même pour les récits parus précédemment dans le Bulletin des Institutrices Catholiques de l'Enseignement Primaire, qu'il regroupe dans son livre Le Renouveau catholique dans l'enseignement primaire. Au même moment, d'autres tirent les leçons de ce mouvement dans les Lettres et Arts, tels Julien Laurec (pseudonyme de l'abbé Julien Le Liboux) (74), l'abbé Calvet (75) ou l'abbé Bremond (76). Les

(65) Jean-Pierre Jossua, Pour une histoire religieuse de l'expression littéraire, t. 2, Paris, Beauchesne, 1990, p. 206.

(66) François Mauriac, Écrits intimes, Paris, 1953, p. 13.

(67) Ce que Michel de Certeau note pour les mystiques du XVI et XVII ${ }^{\mathrm{e}}$ siècles : «L'autobiographie est pour Thérèse d'Avila et bien d'autres avant Descartes, une façon d'“ordonner son âme" et son "esprit" ». Michel de Certeau, La fable mystique, Paris, Gallimard, 1982, p. 166.

(68) Voir à ce titre les essais de Jean-Pierre JossuA, Pour une histoire religieuse de l'expérience littéraire, t. I, II, III et IV. Beauchesne.

(69) Philippe Lejeune, Le pacte autobiographique, Paris, Seuil, 1975, p. 36.

(70) A. Dossat et J. Montjovet, Convertis, Paris, Maison de la Bonne Presse, 1912.

(71) Adolphe RetTÉ, op. cit.

(72) Théodore Mainage, Les témoins du Renouveau catholique, Paris, Beauchesne, 1917, et La psychologie de la conversion. Leçons données à l'Institut catholique de Paris, Paris, Beauchesne, 1919.

(73) Jean-Arthur Chollet, Quelques retours à la foi, Paris, P. Lethielleux, 1931.

(74) Julien Laurec, Le Renouveau catholique dans les lettres, Paris, (1917). Julien Le Liboux (1881-1918) est un assomptionniste. 1927.

(75) Jean CAlvet, Le Renouveau catholique dans la littérature contemporaine, Paris, F. Lanore,

(76) Henri Bremond, dir., Manuel illustré de la Littérature Catholique en France de 1870 à nos jours, Paris, Spes, 1925. 
réflexions de Joseph Huby (77), même si elles tendent, en replaçant les conversions dans une histoire plus longue, à nuancer l'importance du mouvement en ce début de siècle, participent de ce moment d'intérêt soulevé par le phénomène.

Le récit de conversion inscrit les convertis en «médiateurs» entre un catholicisme en crise et une société sécularisée. Il marque la présence et la persistance du sacré au cœur du $\mathrm{XX}^{\mathrm{e}}$ siècle.

\section{Créateurs et médiateurs}

Les convertis s'appuient sur le développement de réseaux d'amitiés littéraires puis spirituelles qui créent les conditions d'éclosion du phénomène et permettent d'acquérir position intellectuelle et lieux de pouvoir qui pérennisent le mouvement. Si les premiers convertis des années 1880-1890 sont isolés, ils offrent en revanche à ceux qui les suivent un soutien qui se traduit dans nombre de cheminements. L'amitié, souvent littéraire au départ, devient ainsi un vecteur de la foi. Les œuvres des convertis participent de leur influence, des milieux se constituent pour accueillir et favoriser les parcours vers le catholicisme. L'Église ne disposant pas d'une structure propice à l'accueil des intellectuels, les rapports d'amitié jouent un rôle décisif. Les convertis se révèlent souvent d'ardents convertisseurs. Claudel, par exemple, engrange les succès pour le ciel (78). De Francis Jammes dans les années 1900 à Maxime Alexandre dans les années 1950, en passant par Jacques Rivière, bien des convertis avouent leur dette de foi au poète.

Les convertis d'avant 1915 jouent aussi un rôle actif dans le recrutement de nouvelles âmes. Les liens d'amitié favorisent la diffusion du phénomène. L'amitié littéraire devient partage de foi. Francis Jammes partage avec Gabriel Frizeau et Charles de Bordeu ses lectures, ses passions intellectuelles mais aussi son cheminement vers la foi. Les Maritain jouent, en particulier dans l'entre-deux-guerres, un rôle important dans ce renouveau du phénomène de conversion. Le «foyer de Meudon» offre un lieu d'accueil qui n'est pas sans réussite. Mais les bruyants échecs autour de la personne de Cocteau et les modifications du champ intellectuel limitent fortement le mouvement.

Les conversions rebondissent de l'un à l'autre, entremêlant amitiés et cheminements personnels. La conjonction entre une interjection, celle de Dieu - du moins selon les convertis - et une invitation, celle des amis, se combinent pour créer les conditions favorables à l'aboutissement d'un parcours ou à sa révélation. Certains membres du clergé ont aussi un rôle indéniable. C'est l'exemple de l'Abbé Mugnier, du Père Clérissac et du Père Janvier. Mais leur rôle est surtout décisif dans l'achèvement des conversions. Des milieux apparaissent donc favorables.

(77) Joseph Huby, La conversion, Paris, Beauchesne, 1919. Ouvrage paru sous forme d'articles dans Études en 1918.

(78) Frédéric Gugelot, «De l'amitié littéraire à l'amitié spirituelle : Claudel convertisseur », in Michel Rouche, dir., Clovis histoire et mémoire, Paris, Presses de l'Université de Paris-Sorbonne, 1997, pp. 615-625. 
Alors que la conversion entraîne parfois de véritables drames familiaux, les convertis retrouvent une nouvelle famille dans l'Église grâce au parrainage. Ainsi Léon Bloy, parrain des Maritain et des Van der Meer de Walcheren les unit par le parrainage des enfants des Van der Meer par les Maritain. Une véritable paternité spirituelle se transmet ainsi à travers les générations (79).

Mais ce lien ne suffit pas. Les convertis cherchent par d'autres structures à encadrer et soutenir leur foi. Et n'est-ce pas la preuve a contrario de leur isolement? «Tout catholique aujourd'hui qui veut garder sa foi au milieu de l'indifférence, sinon de l'hostilité générale, est un combattant (au sens de résistance) et un isolé. C'est de quoi ma vie passée sur les grandes routes m'a donné un sentiment très profond. Lui-même et la société au milieu de laquelle il est appelé à vivre sont guidés par des principes de plus en plus différents, pour ne pas dire antagonistes. - Ce fait est doublement vrai pour un intellectuel (je prends ce mot faute d'un autre). - Il l'est triplement pour un converti, que l'accident qui lui est survenu met brusquement en dehors des relations et d'habitudes souvent anciennes » (80). Paul Claudel, Georges Dumesnil, Gabriel Frizeau, Charles Henrion et Francis Jammes créent en 1910 une Coopérative de prières. «Une conversion est un changement violent qui conduit le converti dans un monde nouveau où il peut se trouver d'abord étonné et dépaysé. Tout en laissant au prêtre le rôle de cure et de direction qui appartient à lui seul, les membres de l'union seront pour le converti une compagnie dans sa nouvelle voie, une famille spirituelle toute formée autour de lui, un corps où il prend sa place, des frères qui se trouvent avec lui sur un pied d'égalité, à qui il peut tout dire, et qui, ayant passé par le même chemin que lui, l'encouragent de leur exemple et l'entraînent de leur mouvement» (81). Un bulletin fait le lien entre les membres. Des prières sont demandées aux différents participants.

Le tiers-ordre connaît aussi un vif succès. Il permet de renforcer sa foi tout en développant toute une sociabilité où le converti sort de son isolement. Les dominicains et les bénédictins connaissent les faveurs de ces nouveaux chrétiens. Les convertis sont plus attirés par les religieux que par les séculiers, ils retrouvent chez eux un identique sentiment d'exil (intérieur ou réel) et surtout un même élitisme. Les néophytes, auxquels leur zèle vaut parfois bien des critiques dans les rangs de l'Église, n'hésitent pas à stigmatiser le clergé et les catholiques dont la «tiédeur» les choque parfois. Certains vont jusqu'au bout de cet itinéraire par une entrée au sein de l'Église. Les monastères attirent. Aux échecs de Huysmans et Claudel répondent l'entrée dans les ordres de Félix Leseur, Jacques Froissart, Jean de Menasce, Maxime Jacob ou, à la fin de leur vie de Louis Massignon et Jacques Maritain. Ainsi, l'Église ne trouve pas dans ces convertis que des plumes ou des palettes à son service, elle y recrute aussi.

Pour favoriser leur implantation dans le monde intellectuel et l'expression de façon plus large de leurs idées, des convertis essayent d'investir bien des positions du champ intellectuel. Rendant responsable de la perte de leur foi l'apologétique de

(79) Frédéric Gugelot, «Un réseau de conversion au catholicisme, Léon Bloy et les siens », in Jean-Dominique DuRAND, dir., La conversion, Association Française d'Histoire Religieuse Contemporaine, Arras, Presses Universitaires d'Artois,1996, pp. 85-94.

(80) Lettre de Paul Claudel à Francis Jammes du 26 mai 1911, Paul Claudel, Francis JAMmEs et Gabriel FrizeAU, op. cit., p. 209.

(81) Lettre de Paul Claudel du 22 septembre 1909, ibid., p. 166. 
leur enfance, ils œuvrent à sa renaissance. La production de récits de conversion participe de cette tentative. Mais cet engagement va plus loin (82). Ils créent en catholiques, reniant souvent leurs écrits antérieurs. Ainsi on peut repérer deux pans bien distincts dans leurs œuvres. C'est le cas par exemple de Joris-Karl Huysmans, d'Adolphe Retté, d'Henri Ghéon. Les thèmes abordés, les choix d'écriture, montrent des évolutions qui apparaissent porteuses d'avenir pour le catholicisme. Non seulement les thèmes de l'œuvre, mais aussi sa facture peuvent changer : le sculpteur Henri Charlier fait le choix de la taille directe pour retrouver la force de l'art médiéval. Charles Du Bos et François Mauriac, après bien d'autres, s'interrogent au début des années 1930 sur les devoirs et les problèmes du romancier catholique. La conversion ouvre des interrogations nouvelles sur leur devenir intellectuel. Se conjuguent alors pour bien des créateurs - peut-être en bout de course -, redécouverte d'un nouveau champ de production et offre d'une production plus moderne à un catholicisme en quête de lui-même. Néanmoins, la conversion réoriente souvent, en parallèle avec leur engagement politique, vers un certain retour à l'ordre esthétique et littéraire, les audaces disparaissent souvent.

L'ambiguïté de leur comportement frappe leurs contemporains. L'abbé Julien Le Liboux, sous pseudonyme, dénonce ces critiques d'où qu'elles viennent : «La publicité qu'ils donnent à leur retour au catholicisme importune nos journalistes libres-penseurs (...) Toujours prompts à suspecter la pureté d'intention de leurs adversaires et incapables de sentir la noblesse des motifs de certains actes qui les dépassent, ils y dénoncent de l'ostentation intéressée, une fructueuse réclame pour les livres prochains du converti. Et ces méchancetés (...) trouvent, hélas! trop souvent créance, parce qu'elles portent coup, par ricochet, contre l'Église, assez inintelligente pour s'applaudir de telles recrues » (83). L'auteur montre bien le lien existant entre la figure du converti et la représentation de l'Église même. Il a aussi conscience que de vives remarques viennent de son propre camp : «Il y a les apologistes quelque peu réclamiers, trop empressés à exploiter la conversion, avant même qu'elle soit accomplie, à chercher là un argument populaire, qu'ils estiment plus impressionnant que de hautes raisons théologiques. Comment voulez-vous qu'un néo-converti, surtout s'il est homme de lettres, ne se laisse pas un peu griser par cette attention violente qui va le chercher?»(84). Aragon frappe plus fort: «Outre le fracas toujours exploité des conversions, on peut estimer qu'honnêtement les convertis sont brusquement séduits par une conciliation offerte de ce qui constitue leur vie (...) et de l'idée qu'ils voudraient donner d'eux-mêmes» (85). L'exploitation de la conversion est donc l'œuvre de tous, convertis comme apologistes. Elle permet une sacralisation de la littérature et conduit à un élitisme perceptible au sein de bien des cheminements vers la foi.

Une indéniable nostalgie spirituelle habite ces chercheurs de foi. C'est un combat culturel qu'ils mènent. L'acte personnel qu'est toute conversion réactive une mémoire à la fois individuelle, d'où la très forte prégnance du souvenir de la mère

(82) Frédéric Gugelot, «Une nouvelle foi, de nouvelles œuvres?», Mélanges de science religieuse, t. 56, 1999, no 1, pp. 49-59.

(83) Julien Laurec, op. cit., p. XII.

(84) Lucien Roure, «La conversion», Études, t. 146, 1916, p. 310.

(85) Louis Aragon, Traité du style, Paris, Gallimard, 1928, p. 101. 
et de la foi d'enfance, et collective, celle d'une tradition (86), celle du catholicisme et de l'Église très vite présentée comme celle d'une patrie, la France. Cette évolution, un journaliste croit la déceler chez Barrès : «L'évolution intellectuelle qu'il a subie devait logiquement le conduire là; on l'y voyait venir. (...) Mais ce fut l'affaire Dreyfus, je crois, qui le révéla à lui-même (...). Il prit nettement et avec fureur parti pour l'iniquité et - peut-être était-il de bonne foi - se lança à toute volée dans le nationalisme, entra à l'Académie française, redevint député, célébra le culte des morts, lança son appel pour la conservation des églises, ne parla plus que d'ancêtres et finalement revint à la foi de ses pères » (87). Que cette annonce soit fausse importe peu, c'est la logique de l'itinéraire ici dévoilée qui lie une certaine vision de la France, des milieux littéraires, et une expression religieuse, le catholicisme.

Le mouvement s'appuie sur la remise en cause du positivisme dominant et sur une vision élitiste du monde intellectuel refusant les évolutions qui se produisent en son sein à la fin du XIX ${ }^{\mathrm{e}}$ siècle. L'encombrement des carrières, la massification et la démocratisation du monde intellectuel (88) conduisent certains à privilégier des lieux d'expression, des modes d'intervention qui leur permettent de se différencier (89). Les affrontements de la Séparation confirment, à leurs yeux, la décadence de la France. Ces convertis mêlent conceptions réactionnaires du monde et catholicisme intégral et ne sont d'ailleurs pas insensibles à l'alchimie entre l'Action française et le catholicisme (90). Se convertir revient à faire scandale par un procédé inédit qui prend le contre-pied de l'établissement dominant.

Ils participent donc des avant-gardes culturelles du début du siècle et introduisent au sein de l'Église, non sans difficultés, de nouvelles formes esthétiques qui offrent du catholicisme une image renouvelée (91). La résistance et les assauts menés contre l'idéologie dominante et les figures intellectuelles du temps (Renan) participent de leur lutte pour ouvrir de nouveaux champs à leur création, pour bénéficier de places au sein d'un monde intellectuel encombré en ce début de siècle. Isabelle Rivière le note très bien quand elle rappelle: «Les jeunes gens d'aujourd'hui (...) ne peuvent imaginer dans quel désert spirituel s'est formée notre jeunesse à nous, qui naissions vers la fin du siècle dernier. Ce n'était pas seulement des petites et grandes Écoles ou Sorbonnes officielles que Dieu était absent; il semblait l'être de partout. Le Grand-Pontife de la philosophie était Auguste Comte, le Grand-docteur des questions religieuses, Ernest Renan, le Grand-Maître de la littérature académique, Anatole France; les révolutionnaires étaient les Symbolistes, chantres d'un monde entièrement païen»(92). Entre cette phrase de 1949 et le

(86) L'acte de croire s'appuie donc sur l'autorité de la tradition. Danièle Hervieu-LÉGER, La religion pour mémoire, Paris, Cerf, 1993, p. 110 (cf. Arch. 84.47).

(87) Paul Brulat, "Conversions religieuses», L'événement, Samedi $1^{\mathrm{er}}$ juin 1912, $1^{\mathrm{èr}}$ page, 2 colonnes à la une.

(88) Christophe Charle, Histoire sociale de la France au XIXe, Paris, Seuil, 1991, pp. 271-273.

(89) Processus déjà amorcé lors de l'Affaire : Christophe CHARLE, "L'affaire Dreyfus en littérature : Bourget et Zola, prophètes et « sociologues » », Paris Fin de siècle, Paris, Seuil, 1998, pp. 201-226.

(90) Jacques PrÉvotat, Les Catholiques et l'Action française, Paris, Fayard, 2001.

(91) Patrick AllitT a aussi montré à quel point les convertis dans le monde anglo-saxon sont aussi les avocats d'un catholicisme qui refuse d'être assimilé à l'obscurantisme, op. cit., p. 1.

(92) Isabelle RIVIĖRE, «Un endroit sacré », Les Bénédictines de la rue Monsieur, Jouques, Éditions du Cloître, 1988, p. 68. 
début de siècle décrit par Isabelle Rivière, se situe toute l'actualité du converti. Ce combat se pare de références et d'appels aux grands ancêtres, que Péguy résume en une phrase : "Qui avait pris le parti de ses pères contre son père, tout est là, tout est dit, et tel est le programme et la dure destinée de notre génération » (93). En reprenant à son compte cette citation tirée d'un ouvrage de Psichari (L'Appel des armes, Paris, Oudin et Cie, 1913 (rééd. Conard, 1919 et 1945)), il justifie la légitimité de sa révolte : «C'est nous ainsi qui sommes l'autorité la plus ancienne et la plus légitime, c'est nous qui sommes la tradition, c'est nous qui sommes la continuité, c'est nous qui sommes les pères, véritablement puisque c'est nous qui sommes la race, puisque c'est nous qui sommes les grands-pères et les aïeux. C'est nous qui sommes la plus haute autorité. C'est nous qui sommes le plus vieux droit (...) C'est nous qui sommes leurs pères. Et ils sont de bien mauvais fils» (94). Ce nouvel élitisme se justifie donc par appel à la tradition et prétend renouer avec elle, isolant et rejetant la légitimité de ceux qui alors dominent le champ intellectuel.

La sécularisation de la société, la privatisation de la foi, le sentiment de persécution qui habite alors le catholicisme conduisent à favoriser la mise en exergue de la figure du converti. La postérité de Péguy dans les années 1930, alors qu'il n’a pas achevé son parcours vers le catholicisme, prouve son actualité. Leur volonté de se rattacher au passé pour mieux justifier leur propre parcours masque la modernité de leur cheminement, et son signe, «l'autonomie du sujet-croyant» (95), et permet leur promotion.

Les convertis sont à l'origine de la création de revues. Joseph Lotte avant la guerre de 1914 crée le Bulletin des professeurs catholiques de l'université. Georges Dumesnil et Robert Vallery-Radot (96) développent au même moment L'Amitié de France. En revanche, la conversion de nombreux membres de la Nouvelle Revue Française d'avant 1914 (Jacques Rivière, Henri Ghéon, Jacques Copeau...) ou de nombreux auteurs de la maison (Claudel, Larbaud...) ne permet pas de faire de la $N R F$ une revue catholique. D'autres tentatives comme celle de Vigile, autour de Charles du Bos et François Mauriac, au début des années trente échouent elles aussi. Les convertis $\mathrm{y}$ trouvent néanmoins des tribunes, aussi éphémères soient-elles.

Après guerre, le théâtre est un des champs d'action le plus vivant des convertis. Henri Ghéon et Jacques Copeau, convertis respectivement en 1915 et 1926, tentent de donner à un théâtre chrétien un nouveau souffle. L'idée reste la même, renouveler la défense et la diffusion du message de Dieu. Leur volonté d'ouvrir au plus grand nombre l'accès à la culture, une culture religieuse s'entend, n'est pas sans avenir.

Mais surtout des convertis obtiennent dans le monde de l'édition des places leur permettant de développer de nouvelles collections catholiques. Le Roseau

(93) Charles PÉguy, L'Argent, ceuvres en prose complètes, t. III, Paris, Gallimard, 1992, p. 900.

(94) Ibid., p. 900.

(95) Danièle HERVIEU-LÉGER, op. cit., p. 121.

(96) Hervé SERRY, «Le mouvement de «renaissance littéraire catholique» : entre espoirs et désillusions », in Francis Jammes et Robert VALLERY-RAdot, Correspondance (1906-1934), Bulletin de 1'association Francis Jammes, $\mathrm{n}^{\circ}$ 28, déc. 1998, pp. 11-50; «L'artiste et le sacré. J. Maritain et l'affaire de la Jeanne d'Arc de J. DelteIl », Cahiers Jacques Maritain, no 36, juin 1998, pp. 15-39 et «Les écrivains catholiques dans les années $20 »$, Actes de recherche en sciences sociales, n 124, sept. 1998, pp. 80-87. 
d'Or (97), paru chez Plon de 1925 à 1933, et dirigé par Maritain et Stanislas Fumet, s'enorgueillit de son catalogue. À la Correspondance entre Jacques Rivière et Paul Claudel s'ajoutent la première journée du Soulier de Satin, Le gant de crin de Pierre Reverdy, Un nouveau Moyen Âge de Berdiaeff et les ouvrages de Georges Bernanos (Sous le soleil de Satan, expurgé, il est vrai), Henri Ghéon, Julien Green, Henri Massis et Jacques Maritain. Si le Roseau d'Or apparaît comme un succès, d'autres tentatives sont plus limitées. Ainsi La NEF chez Spes, qui souhaite exprimer les traits caractéristiques de la renaissance littéraire catholique. Néanmoins ces nouveaux chrétiens trouvent par là des lieux d'édition et donc de diffusion de leur tentative d'évangéliser l'intelligence.

Les convertis apportèrent à l'Église et au catholicisme la vivacité de leur jeune adhésion et l'effet de leur appartenance à des milieux considérés comme éloignés de la foi. Leur importance vient en effet aussi de ce qu'ils sont à contre courant. Ils ne furent pas alors sans influence sur le renouveau catholique des années 1930 et sur les futures évolutions de l'Église. À la fin de ces années, les conversions ne s'interrompent pas mais la mise en représentation de ce type de cheminement n'est plus nécessaire. L'Église ne se perçoit plus comme une citadelle assiégée à qui ces écrivains, artistes et musiciens revenus en son sein donnent les moyens de prouver qu'elle n'est pas qu'obscurantisme. Si «la conjoncture spécifique des années 1930, ecclésiale et française, a accouché (...) de la catégorie "intellectuels catholiques"» (98), cette victoire s'inscrit sur la disparition du converti. Ils ne sont plus les hérauts dont elle a besoin.

De la défensive, les catholiques sont passés à l'offensive. Il s'agit de conquérir ou de reconquérir les milieux éloignés. "Refaisons chrétiens, nos frères», lance, peu après la Jeunesse ouvrière chrétienne. Une «nouvelle chrétienté» est à bâtir (99). L'individu s'estompe au profit des masses, l'action prime. La vigueur du mouvement apostolique, en particulier au sein de la jeunesse, témoigne du réveil du catholicisme français. Dans le même temps, œcuménisme et liberté religieuse font des progrès, poussent à l'extinction ou à l'atténuation progressive des différends religieux et tendent à estomper - pour un temps? - le flot des conversions. Ou du moins leur promotion au sein de l'Église.

\section{Frédéric GUGELOT}

Université de Reims

(97) Philippe Chenaux, Entre Maurras et Maritain, Paris, Cerf, 1999.

(98) Étienne Foullloux, " "Intellectuels catholiques”. Réflexion sur une naissance différée », Vingtième siècle, no 53, janv.-mars 1997, p. 22.

(99) Étienne Foullloux, «Une brillante réponse», in René RÉmond, dir., Histoire de la France religieuse, t. 4, xx ${ }^{\mathrm{e}}$ siècle, Paris, Seuil, 1992, p. 215. 


\section{Résumé}

Dans un temps, les années 1910, où de sécularisation et de désenchantement, la figure du converti fut promue grâce au récit de conversion. Le converti était la preuve de la présence de Dieu au XX siècle et de la pérennité de l'Église. Mais il révélait aussi un monde sans foi où la modernité religieuse s'exprimait par l'engagement individuel d'adultes et non par la tradition transmise. Un réseau d'amitié littéraire puis spirituelle permit à ces cheminements personnels de trouver des soutiens tout en amplifiant le mouvement.

\section{Abstract}

The catholic converts among the French intellectuals at the beginning of the $X X^{e}$ century think that they belong to a world without faith. The turn to Rome appears through spiritual autobiographies which unveil litterary and spiritual friendship in the movement and reveals the modernity of such a way of believing.

\section{Resumen}

En un tiempo, los años 1910, de secularización y de desencantamiento, la figura del converso fue promovida gracias al relato de conversión. El converso era la prueba de la presencia de Dios en el siglo XX, y de la perennidad de la Iglesia. Pero también daba cuenta de un mundo sin fe, en el que la modernidad religiosa se expresaba a través del compromiso individual de los adultos y no por la tradición transmitida. Una red de amistad literaria, luego espiritual, permite a estos recorridos personales encontrar sostenes, y al mismo tiempo amplificar el movimiento. 